\title{
IMMERSIONS OF HIGHLY CONNECTED MANIFOLDS
}

\author{
REINHARD WIEGMANN
}

\begin{abstract}
If $\left[M^{n} \rightarrow \mathbf{R}^{2 n-k}\right]$ denotes the set of regular homotopy classes of immersions, $M^{n}$ a $k$-connected compact manifold, we show by a direct geometric construction the correspondence $\left[M^{n} \rightarrow \mathbf{R}^{2 n-k}\right] \rightarrow\left[S^{n} \rightarrow \mathbf{R}^{2 n-k}\right]$ for $0 \leq 2 k \leq n-2$.
\end{abstract}

A bijection $I\left[M^{n} \rightarrow \mathbf{R}^{2 n-k}\right] \rightarrow\left[S^{n} \rightarrow \mathbf{R}^{2 n-k}\right]$ can be obtained as follows: Any immersion $g: M^{n} \rightarrow \mathbf{R}^{2 n-k}$ is regularly homotopic to an immersion $\bar{g}$ with $\bar{g} \mid M \backslash U$ an embedding, where $U$ is a suitable coordinate neighborhood of the basepoint. Set $I([g])=$ the immersion of $S^{n}$ determined by $\bar{g} \mid U$. That $I$ is a bijection follows from $[2,3]$. This paper gives a simple, direct geometric construction of the map $\bar{g}$.

Proposition. $M^{n}$ a compact, closed, $k$-connected manifold. If $0 \leq 2 k \leq n-2$, there is a bijection

$$
I:\left[M^{n} \leftrightarrow \mathbf{R}^{2 n-k}\right] \rightarrow \Pi_{n}\left(V_{n}\left(\mathbf{R}^{2 n-k}\right)\right) .
$$

REMARK. The correspondence $\left[S^{n} \rightarrow \mathbf{R}^{2 n-k}\right] \rightarrow \Pi_{n}\left(V_{n}\left(\mathbf{R}^{2 n-k}\right)\right)$ is a classical result of S. Smale [7]. Immersions of spheres are classified by their double points [4, 6]; this result extends this double point classification to highly connected manifolds.

For the proof, we first recall some well-known facts. By [2] a $k$-connected compact closed manifold $M^{n}$ can be embedded in $\mathbf{R}^{2 n-k}$ if $2 k \leq n-2$. Moreover, two embeddings are regularly homotopic on $M \backslash\left\{x_{0}\right\}$. These results, together with [7], permit the construction of a group action

$$
\left[S^{n} \rightarrow \mathbf{R}^{2 n-k}\right] \times\left[M^{n} \rightarrow \mathbf{R}^{2 n-k}\right] \rightarrow\left[M^{n} \rightarrow \mathbf{R}^{2 n-k}\right]
$$

which is seen to be free and transitive, heise the result. Let $g: M^{n} \rightarrow \mathbf{R}^{2 n-k}$ represent a class $[g] \in\left[M^{n} \rightarrow \mathbf{R}^{2 n-k}\right]$, and $f: S^{n} \rightarrow \mathbf{R}^{2 n-k}$ represent an immersion with Smale invariant $c(f) \in \Pi_{n}\left(V_{n}\left(\mathbf{R}^{2 n-k}\right)\right)$. The connected sum of $g$ and $f$ yields an immersion $f \# g: M^{n} \rightarrow \mathbf{R}^{2 n-k}$, and we define

$$
\begin{gathered}
{\left[S^{n} \leftrightarrow \mathbf{R}^{2 n-k}\right] \times\left[M^{n} \leftrightarrow \mathbf{R}^{2 n-k}\right] \rightarrow\left[M^{n} \leftrightarrow \mathbf{R}^{2 n-k}\right]} \\
([f],[g]) \mapsto[f \# g] .
\end{gathered}
$$

It is obvious that in this way one obtains a group action. If $g$ is as above, we may deform $g$ by a regular homotopy such that

$$
g \times g: M \times M \backslash \Delta_{M} \rightarrow \mathbf{R}^{2 n-k} \times \mathbf{R}^{2 n-k}
$$

is transverse to the diagonal $\Delta \subset \mathbf{R}^{2 n-k} \times \mathbf{R}^{2 n-k}$.

The set $M_{g}=\{x \in M \mid \exists y \in M, x \neq y, g(x)=g(y)\}$ of double points of $g$ is a $k$-dimensional submanifold if $2 k \leq n-1$. The embedding $i: M_{g} \hookrightarrow M$ is null

Received by the editors December 6, 1982.

1980 Mathematics Subject Classification. Primary 57R42; Secondary 55Q52.

(C) 1984 American Mathematical Society $0002-9939 / 84 \$ 1.00+\$ .25$ per page 
homotopic, so we may choose a homotopy $H: M_{g} \times I \rightarrow M$ from $i$ to the trivial map $M_{g} \rightarrow x_{0} \in M$. For a suitable coordinate neighbourhood $U \subset M^{n}$ of $x_{0}$ and a chart $k:\left(D^{n}, 0\right) \rightarrow\left(U, x_{0}\right)$ we can find $\epsilon>0$ such that $H_{\epsilon}\left(M_{g}\right) \subset U$ for $t \in[1-\epsilon, 1]$. For $2 k \leq n-2$ we can approximize $H$ on $[0,1-\epsilon / 2]$ by $\bar{H}$, such that $\bar{H}_{t}: M_{g} \rightarrow M$ is an embedding. By a reparametrization we obtain an isotopy $\bar{H}: M_{g} \times I \rightarrow M$ with $\bar{H}_{0}=i$ and $\bar{H}_{1}\left(M_{g}\right) \subset U$. Since $M^{n}$ is closed, there is a diffeotopy $G: M \times I \rightarrow M$ such that

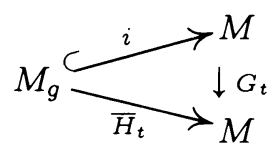

commutes for all $t \in I$.

$g \circ G^{-1}$ is a regular homotopy from $g$ to $\bar{g}$, and $U$ contains the double points $M_{\bar{g}}$ of $\bar{g} . \quad \bar{g}$ restricted to $M \backslash \stackrel{\circ}{U},(\stackrel{\circ}{U}=$ interior of $U)$ is an injective immersion of a compact manifold with boundary, hence an embedding. By [5] the regular homotopy class of $\bar{g}_{\mid M \backslash \cup}$ is unique.

$\bar{g} \circ K_{\mid \partial D^{n}}: S^{n-1} \rightarrow \mathbf{R}^{2 n-k}$ is an embedding with Smale invariant zero, thus regularly homotopic to the standard embedding $S^{n-1} \hookrightarrow \mathbf{R}^{2 n-k}$. Define $\tilde{g}: S^{n} \rightarrow$ $\mathbf{R}^{2 n-k}$ by $\bar{g} \circ k: D^{n} \rightarrow \mathbf{R}^{2 n-k}$ on the northern hemisphere and a standard embedding on the southern hemisphere. Defining $I[g]=c[\tilde{g}]$, where $c$ means take the Smale invariant, we obtain our map $I:\left[M^{n} \rightarrow \mathbf{R}^{2 n-k}\right] \rightarrow\left[S^{n} \rightarrow \mathbf{R}^{2 n-k}\right]$.

Claim. (i) $I$ is well defined. (ii) $I$ is bijective.

(i) Let $f, g$ represent a class in $\left[M^{n} \rightarrow \mathbf{R}^{2 n-k}\right]$. Proceeding as above we can regularly homotope $f$ and $g$ to an embedding outside a suitably chosen coordinate neighborhood of the basepoint of $M$. By [2] we may assume $f|M \backslash U=g| M \backslash U$ and a regular homotopy from $f$ to $g$ may be assumed to be an isotopy on $M \backslash U$. A regular homotopy from $f$ to $g$ is therefore given by a regular homotopy rel $\partial U$ from $f \mid U$ to $g \mid U$. Applying the above gluing construction, one obtains a regular homotopy from $f$ to $g$.

(ii) Let $j: M^{n} \hookrightarrow \mathbf{R}^{2 n-k}$ be an embedding. An inverse $J$ to $I$ is given by $J(\varphi)=$ $f_{\varphi} \# j$, where $f_{\varphi}: S^{n} \rightarrow \mathbf{R}^{2 n-k}$ is an immersion with Smale invariant $\varphi$.

\section{REFERENCES}

1. A. Haefliger, Plongements differentiables des variétés dans variétés, Comment. Math. Helv. 36 (1962).

2. A. Haefliger and M. W. Hirsch, On the existence and classification of differentiable embeddings, Topology 2 (1963).

3. M. W. Hirsch, Immersions of manifolds, Trans. Amer. Math. Soc. 93 (1959), 242-276.

4. U. Koschorke and B. Sanderson, Geometric interpretation of the generalized Hopf invariant, Math. Scand. 41 (1977).

5. M. Kervaire, Sur l'invariant de Smale d'un plongement, Comment. Math. Helv. 34 (1960).

6. C. Olk, Immersionen von Mannigfaltigkeiten in euklidische Räume, Dissertation, Siegen, 1980.

7. S. Smale, The classification of immersions of spheres in Euclidean space, Ann. of Math. (2) 69 (1959), 327-344.

Department of Mathematics, Universität Osnabrück, 4S Osnabrück, Postfach 4469, West Germany 